\title{
Weight Functions for Chebyshev Quadrature
}

\author{
By Yuan Xu
}

\begin{abstract}
In this paper, we investigate if the weight function $\left(1-x^{2}\right)^{-1 / 2} R(x)$, where $R(x)$ is a rational function of order $(1,1)$, admits Chebyshev quadratures. Many positive examples are provided. In particular, we have proved that the answer is affirmative if $R(x)=1+b x,|b|<0.27846 \ldots$
\end{abstract}

1. Let $w$ be an integrable nonnegative weight function on $[-1,1]$. A Chebyshev quadrature formula is an equally weighted quadrature

$$
\int_{-1}^{1} f(x) w(x) d x=A_{n} \sum_{k=1}^{n} f\left(x_{k}\right)+R_{n}[f]
$$

with real distinct nodes $x_{k} \in(-1,1)$ and algebraic degree of exactness $n$, i.e., $R_{n}[f]=0$ for every $f \in \Pi_{n}$, the class of polynomials of degree $\leq n$. If an $n$-point Chebyshev quadrature formula exists for every positive integer $n$, we say that the weight function $w$ has property $T$.

It is well known that the classical weight function $w_{0}(x)=(1 / \pi)\left(1-x^{2}\right)^{-1 / 2}$ has property $T$ and in fact produces an equally weighted Gaussian quadrature formula, but weight functions with property $T$ are rare. (For a recent survey of this topic, see Gautschi [3].) The first example other than $w_{0}$ is due to Ullman [8]. He proved that

$$
w(x)=w_{0}(x) \frac{1+p x}{1+p^{2}+2 p x}, \quad|p|<\frac{1}{2},
$$

gives an infinite one-parameter family of weight functions having property $T$. In a more recent paper, Byrd and Stalla [1] provided another class of weight functions with property $T$,

$$
w(x)=w_{0}(x) \frac{1}{2 p+1+x}, \quad p \geq 1 .
$$

Further examples and related results can be found in [2], [4], [5], and [7]. But the problem of characterizing all weight functions with property $T$ is still open, and an attempt of finding the complete solution to this problem would seem to be too ambitious. The most difficult part is investigating the zeros of the polynomials that provide the nodes.

Now consider the weight function

$$
w(x)=w_{0}(x) \frac{(1+a b)+(a+b) x}{1+a^{2}+2 a x}, \quad-1 \leq x \leq 1 .
$$

Received October 17, 1987; revised March 1, 1988 and July 20, 1988.

1980 Mathematics Subject Classification (1985 Revision). Primary 65D32.

Key words and phrases. Chebyshev quadrature, weight function with property $T$. 
Since the substitution $a \mapsto a^{-1}, b \mapsto b^{-1}$ leaves this weight function invariant (up to a constant factor), it is easily checked that (4) is a positive weight function not equal to a constant multiple of $w_{0}$ if and only if

$$
|a|<1 \quad \text { and } \quad|b|<1 \text {. }
$$

We may thus assume (5).

For weight functions (4), Geronimus and Medvedeva [5] showed that there exists an infinite sequence of Chebyshev quadrature formulas for each fixed rational $b / a$, if $0<b / a<1$. From a general result of Peherstorfer [7, Theorem 2(b)] $(\Phi:=$ $(a-b) /(1+b z))$, it also follows that for all $a, b$ satisfying

$$
|a-b| \leq(1 / 8)-|b|, \quad|b| \leq 1 / 8
$$

the respective weight functions $w$ have property $T$.

The main results of this paper are the following.

THEOREM 1. The weight function (4) has property $T$ if one of the following conditions is satisfied:

(i) $b=0,|a|<1 / 2$,

(ii) $1>b / a>0,0<|a| \leq 1 / 3$,

(iii) $b / a<0,[(1-|a|) / 2]^{1-b / a}>|a|, 0<|a| \leq 1 / 3$,

(iv) $[(1-|a|) / 2]^{1-b / a}>|a|\{(1 / 2)(1+|a|) /(1-|a|)\}^{1 / 2}, 1 / 3 \leq|a|<1, b / a<1$.

THEOREM 2. The weight function $w(x)=w_{0}(x)(1+b x)$, i.e., (4) in the case of $a=0$, has property $T$ if $|b|<q$, where $q=0.2784645 \ldots$ is the root of $\exp (-x)=e x$.

Remark 1. When $a=b$, then $w(x)=w_{0}(x)$. When $b=0$, Theorem 1 (i) gives Ullman's result (2). When $b=-a$, Theorem 1 (iii) gives Byrd and Stalla's result (3) with condition $|a|<3-2 \sqrt{2}$, which is exactly $p \geq 1$ in (3).

Remark 2. We list a separate theorem for the case $a=0$, because it is interesting to note that even the product of $w_{0}(x)$ with a linear function can give an infinite family of weight functions with property $T$.

Remark 3. Kahaner [6] has shown that for $w(x)=w_{0}(x) v(x), v$ continuous in $(-1,1)$, to admit Chebyshev quadrature, a necessary condition on $v$ is

$$
v(x) \geq \frac{1}{2} \int_{-1}^{1} w(x) d x / \int_{-1}^{1} w_{0}(x) d x \quad \text { for all } x \in(-1,1) .
$$

Therefore, the weight function $w(x)=w_{0}(x)(1+b x)$ does not have property $T$ if $|b|>1 / 2$.

2. Let $p_{n}(w, x)=\Pi\left(x-x_{k}\right)$, where the $x_{k}$ are the nodes in quadrature formula (1). By a formula due to Chebyshev, $p_{n}(w, x)$ can be represented explicitly in the form

$$
p_{n}(w, x)=E\left\{\exp \left(n \int_{-1}^{1} w(t) \ln (x-t) d t\right)\right\}
$$

where $E\{\cdot\}$ denotes the polynomial part of $\{\cdot\}$. Based on this formula, Geronimus [4] gave the following result.

If we consider the weight function in trigonometric form,

$$
w(x)=w(\cos \phi)=\frac{1}{\pi \sin \phi} \sum_{k=0}^{\infty} a_{k} \cos k \phi, \quad 0 \leq \phi \leq \pi, a_{0}=1,
$$


and denote

$$
\exp \left[-n \sum_{k=1}^{\infty} a_{k} z^{-k} / k\right]=\sum_{m=0}^{\infty} A_{m}^{(n)} z^{-m}, \quad A_{0}^{(n)}=1,|z|>1
$$

then

$$
2^{n-1} p_{n}(w, x)=\sum_{k=0}^{n-1} A_{k}^{(n)} T_{n-k}(x)+A_{n}^{(n)} / 2,
$$

where $T_{m}(x)$ is the $m$ th-degree Chebyshev polynomial of the first kind.

We now use this method to form $p_{n}(w, x)$ in our case. Expanding (4) into a Chebyshev-Fourier series, it follows from $[4$, p. 1118] that

$$
A_{k}^{(n)}=\left(\begin{array}{c}
\alpha n \\
k
\end{array}\right) a^{k}
$$

where $\alpha=1-(b / a)$. Therefore we get from (8) and (9)

$$
2^{n-1} p_{n}(w, x)=\sum_{k=0}^{n-1}\left(\begin{array}{c}
\alpha n \\
k
\end{array}\right) a^{k} T_{n-k}(x)+\left(\begin{array}{c}
\alpha n \\
n
\end{array}\right) a^{n} / 2, \quad a \neq 0 .
$$

If $a=0$, we have by (6),

$$
\exp \left[-n \sum_{k=1}^{\infty} a_{k} z^{-k} / k\right]=\exp (-n b / z)=\sum_{k=0}^{\infty}(-b n / z)^{k} / k !
$$

From (7) and (8), we obtain for $a=0$

$$
2^{n-1} p_{n}(w, x)=\sum_{k=0}^{n-1}(-b n)^{k} T_{n-k}(x) / k !+(1 / 2)(-b n)^{n} / n !
$$

If all the zeros of $p_{n}(w, x)$ are distinct and in $(-1,1)$, for all $n \geq 1$, then $w$ has property $T$ (see, e.g., [3]). So we only need to investigate the zeros of (10) and (11).

3. In this section we assume $a \neq 0$ and prove Theorem 1. First we rewrite $p_{n}(w, x)$. By

$$
(a+\exp (i \phi))^{\alpha n}=\sum_{k=0}^{\infty}\left(\begin{array}{c}
\alpha n \\
k
\end{array}\right) a^{k} \exp [i \phi(\alpha n-k)],
$$

we get from (10) that

$$
\begin{aligned}
2^{n} p_{n}(w, x)= & 2 \operatorname{Re}\left[\exp (-i n \phi)[1+a \exp (i \phi)]^{\alpha n}\right] \\
& -\left[2 \sum_{k=n+1}^{\infty}\left(\begin{array}{c}
\alpha n \\
k
\end{array}\right) a^{k} \cos (n-k) \phi+\left(\begin{array}{c}
\alpha n \\
n
\end{array}\right) a^{n}\right] .
\end{aligned}
$$

Let

$$
\exp (-i \phi)[1+a \exp (i \phi)]^{\alpha}=R \exp (i \psi)
$$

we then have

$$
2^{n} p_{n}(w, x)=2 R^{n} \cos n \psi-J,
$$

where $J=J(\phi)$ is the second term in the right-hand side of (13),

$$
R=|1+a \exp (i \phi)|^{\alpha}=\left(1+a^{2}+2 a \cos \phi\right)^{\alpha / 2},
$$


and

$$
\begin{aligned}
\cos \psi & =\operatorname{Re}\left[\exp (-i \phi)[1+a \exp (i \phi)]^{\alpha}\right] / R \\
& =\left(x+\sum_{k=1}^{\infty}\left(\begin{array}{l}
\alpha \\
k
\end{array}\right) a^{k} T_{k-1}(x)\right) /\left(1+a^{2}+2 a x\right)^{\alpha / 2}, \quad x=\cos \phi .
\end{aligned}
$$

It is easy to check that if $0 \leq \phi \leq \pi$, then $0 \leq \psi \leq \pi$, and $\psi=0$ for $\phi=0$, $\psi=\pi$ for $\phi=\pi$. Since $\psi$ is a continuous function of $\phi$, there exists a sequence $\phi_{k}$, $0 \leq k \leq n, \phi_{0}<\phi_{1}<\cdots<\phi_{n}$, with

$$
\psi\left(\cos \phi_{k}\right)=\psi_{k}=k \pi / n \text {. }
$$

(It can be proved that $\psi$ is a monotone function of $\phi$; therefore $\left\{\phi_{k}\right\}$ is uniquely defined by (17).) Since $\cos n \psi_{k}=(-1)^{k}$, we get from (14) that $p_{n}(w, x)$ will change sign exactly $n$ times in $(-1,1)$ if

$$
2 R^{n}=2\left(1+a^{2}+2 a \cos \phi\right)^{\alpha n / 2}>|J|
$$

for $0 \leq \phi \leq \pi$.

If $b=0$, i.e., $\alpha=1,(18)$ is just $2 R^{n}>|a|^{n}$. It is easy to check that this is true for any $n$ and $\phi$ if $|a|<1 / 2$. Actually, this is Ullman's case (2). If $\alpha \neq 1$, we first estimate $J$. By the Residue Theorem,

$$
\left(\begin{array}{c}
\alpha n \\
k
\end{array}\right) \varepsilon^{k}=\frac{1}{2 \pi i} \int_{|z|=1}(1+\varepsilon z)^{\alpha n} / z^{k+1} d z,
$$

where $|a|<\varepsilon<1$, so that for $\alpha>0$,

$$
\begin{aligned}
|J| & \leq \frac{1}{2 \pi}\left|\frac{a}{\varepsilon}\right|^{n} \int_{|z|=1}|1+\varepsilon z|^{\alpha n}\left[2 \sum_{k=0}^{\infty}(|a| / \varepsilon)^{k+1}+1\right]|d z| \\
& \leq(1+\varepsilon)^{\alpha n}|a / \varepsilon|^{n}(\varepsilon+|a|) /(\varepsilon-|a|),
\end{aligned}
$$

where the legitimacy of interchanging sum and integral can be easily checked. Letting $\varepsilon \rightarrow 1$, we get

$$
|J| \leq 2^{\alpha n}|a|^{n}(1+|a|) /(1-|a|) .
$$

Therefore (18) holds if

$$
2 R^{n}=2\left(1+a^{2}+2 a x\right)^{\alpha n / 2}>2^{\alpha n}|a|^{n}(1+|a|) /(1-|a|)
$$

for any $-1 \leq x \leq 1$. Hence, the existence of an $n$-point Chebyshev formula follows if

$$
[(1-|a|) / 2]^{\alpha}>|a|[(1+|a|) / 2(1-|a|)]^{1 / n} .
$$

If $|a| \leq 1 / 3$, we have that (19) holds if

$$
[(1-|a|) / 2]^{\alpha}>|a| \text {. }
$$

Notice that $[(1-|a|) / 2]^{\alpha}$ is a decreasing function of $\alpha$, and for $\alpha<1,(20)$ gives $|a| \leq 1 / 3$. We therefore have proven (ii) and (iii). If $|a| \geq 1 / 3$, inequality (19) is satisfied for $n \geq 2$ if

$$
[(1-|a|) / 2]^{\alpha}>|a|[(1+|a|) /(2-2|a|)]^{1 / 2} .
$$

Note that for $n=1$ the Chebyshev formula always exists, since this is the 1-point Gauss formula. Therefore, the proof is completed. 
4. Now we assume $a=0$ and prove Theorem 2. First rewrite $p_{n}(w, x)$ as in the last section. Let $x=\cos \phi$; then by

$$
\sum_{k=0}^{\infty}(-b n \exp (-i \phi))^{k} / k !=\exp [-b n \exp (-i \phi)]
$$

from (11) we have

$$
\begin{aligned}
2^{n} p_{n}(w, x)= & 2 \operatorname{Re}\{\exp [i(\phi+b \sin \phi) n] \exp (-b n \cos \phi)\} \\
& -\left[2 \sum_{k=n+1}^{\infty}(-b n)^{k} \cos (n-k) \phi / k !+(-b n)^{n} / n !\right] .
\end{aligned}
$$

Letting

$$
R=\exp (-b \cos \phi), \quad \psi=\psi(\phi)=\phi+b \sin \phi
$$

we get

$$
2^{n} p_{n}(w, x)=2 R^{n} \cos n \psi-J
$$

where $J=J(\phi)$ is the second term in the right-hand side of (21).

Since $|b|<1$, and $\psi(0)=0, \psi(\pi)=\pi$, we know that $\psi(\phi)$ is a continuous function from $[0, \pi]$ to $[0, \pi]$. Therefore by $(23)$, according to the argument in the proof of Theorem $1, p_{n}(w, x)$ will have exactly $n$ distinct zeros in $(-1,1)$ if

$$
2 R^{n}=2 \exp (-b n \cos \phi)>|J|
$$

for any $0 \leq \phi \leq \pi$.

We now estimate $J$. Since $n^{k} n ! /(k+n) ! \leq 1$, we get

$$
\begin{aligned}
|J| & \leq\left[2 \sum_{k=1}^{\infty}(|b| n)^{k} n ! /(k+n) !+1\right](|b| n)^{n} / n ! \\
& \leq\left[2 \sum_{k=1}^{\infty}|b|^{k}+1\right](|b| n)^{n} / n !=\frac{1+|b|}{1-|b|}(|b| n)^{n} / n !
\end{aligned}
$$

So we have

$$
|J| \leq 2(|b| n)^{n} / n ! \quad \text { when }|b| \leq 1 / 3 .
$$

Furthermore, Stirling's formula gives

$$
1 / n !<e^{n} / n^{n}(2 \pi n)^{1 / 2} \text {. }
$$

It then follows from (25) that

$$
|J| \leq 2(|b| e)^{n} /(2 \pi n)^{1 / 2}<2(|b| e)^{n} .
$$

By (24) and (26), we need to consider

$$
\exp (-b n \cos \phi) \geq(|b| e)^{n},
$$

for any $0 \leq \phi \leq \pi$. Therefore (24) holds if

$$
\exp (-|b|) \geq|b| e \text {. }
$$

It is easy to see that $f(x)=\exp (-x)-e x$ is a decreasing function, and the unique solution of $f(x)=0$ is in $(0,1)$. So (27) holds for

$$
|b|<q=0.2784645 \ldots,
$$

where $q$ is the unique solution of $\exp (-x)=e x$. This completes the proof of Theorem 2. 
Acknowledgment. The author wishes to thank Professor W. Gautschi and a referee for the great care with which the details of the paper have been checked, and for many invaluable suggestions.

Department of Mathematics

Temple University

Philadelphia, Pennsylvania 19122

1 P. F. BYRD \& L. STALla, "Chebyshev quadrature rules for a new class of weight functions," Math. Comp., v. 42, 1984, pp. 173-181.

2. K. J. FÖRSTER, "On weight functions admitting Chebyshev quadrature," Math Comp., v. 49, 1987, pp. 251-258.

3. W. GAUTSCHI, Advances in Chebyshev Quadrature, Lecture Notes in Math., vol. 506, SpringerVerlag, Berlin and New York, 1976, pp. 100-121.

4. JA. L. Geronimus, "On the Chebyshev quadrature formula," Izv. Akad. Nauk SSSR Ser. Mat., v. 33, 1969, pp. 1182-1207; English transl. in Math. USSR-Izv., v. 3, 1969, pp. 1115-1138.

5. JA. L. Geronimus \& A. K. Medvedeva, "The validity of the 'Chebyshev's rule' for a certain two-parameter family of weight functions," Dokl. Akad. Nauk SSSR, v. 224, 1975, pp. 516-518; English transl. in Soviet Math. Dokl, v. 16, 1975, pp. 1231-1233.

6. D. K. KAHANER, "On equal and almost equal weight quadrature formulas," SIAM J. Numer. Anal., v. 6, 1969, pp. 551-556.

7. F. PEHERSTORFER, "Weight functions which admit Tchebycheff quadrature," Bull. Austral. Math. Soc., v. 26, 1982 , pp. 29-38.

8. J. L. Ullman, "A class of weight functions that admit Tchebycheff quadrature," Michigan Math. J., v. 13, 1966, pp. 417-423. 